\title{
ATUALIZAÇÃO SOBRE MEDIADORES E MODELOS EXPERIMENTAIS DE SEPSE
}

\author{
PRESENT UNDERSTANDING OF MEDIATORS AND EXPERIMENTAL MODELS OF SEPSIS
}

\author{
Claudia F. Benjamim
}

Pós-doutoranda do Departamento de Farmacologia da Faculdade de Medicina de Ribeirão Preto da Universidade de São Paulo. CoRRESPONDÊnCIA: Dra. Claudia Farias Benjamim. Av. Bandeirantes, 3900 - FMRP - USP - CEP: 14049-900 Ribeirão Preto, SP - Fone: (16) 602-3204 - FAX: (16) 633-2301 - E-mail: benjacau@usp.br

BENJAMIM CF. Atualização sobre mediadores e modelos experimentais de sepse. Medicina, Ribeirão Preto, 34: 18-26, jan./mar. 2001.

RESUMO: A autora apresenta uma revisão sobre a resposta inflamatória durante a sepse, abordando os principais mediadores envolvidos no processo, tais como citocinas e óxido nítrico, bem como o papel desses mediadores na evolução da doença. Esse aspecto abordado não é tão simples e pontual, pois a relevância desses mediadores inflamatórios, na sepse, varia de acordo com muitos fatores, entre eles, com o modelo experimental utilizado, a espécie animal e o estágio da doença. Ainda, serão revisados os modelos experimentais mais utilizados para o estudo da sepse, discutindo-se as vantagens de cada modelo e a sua contribuição para a compreensão da doença. Não há dúvida que os modelos experimentais, utilizados hoje em dia, contribuem bastante para o entendimento de sepse e choque séptico. Entretanto, é importante saber discernir os pontos fortes e fracos do modelo, possibilitando uma real extensão dos resultados experimentais para testes clínicos.

UNITERMOS: Sepse. Choque Séptico. Endotoxinas. Peritonite. Lipopolissacarídios. Citocinas.

\section{INTRODUÇÃO}

Em um quadro de sepse, devem ser avaliados e combatidos três processos distintos, porém interligados, que acontecem concomitantemente: o foco infeccioso, as alterações hemodinâmicas e a resposta inflamatória local e generalizada. Até hoje, o tratamento de pacientes com sepse ou choque séptico é feito por meio de antibióticos e drogas que interferem nas alterações cardiovasculares $^{(1)}$, não se intervindo na resposta inflamatória, podendo ser esse um dos motivos da alta mortalidade de pacientes com choque séptico. Ademais, ainda não se demonstrou, inequivocamente, a relativa contribuição de cada mediador inflamatório na letalidade resultante do choque séptico. Dessa forma, é de extrema importância o estudo da resposta inflamatória durante a sepse e este trabalho tem como objetivo descrever os modelos experimentais de sepse mais utilizados para o estudo da evolução da sepse, e revisar os mediadores inflamatórios, envolvidos e que apresentam papel relevante na sepse.

\subsection{Conceitos fundamentais a a fisiopatologia da sepse}

O termo sepse é definido como síndrome da resposta inflamatória, sistêmica (SRIS), decorrente de infecção, principalmente por bactérias, embora, também, possa ser consequiência de infecção por fungos, helmintos e vírus. As infecções causadas por bactérias gram-negativas são as mais freqüentes, apesar de, na última década, ter havido um aumento de casos de sepse devido a bactérias gram-positivas. A definição de SRIS inclui tanto a sepse quanto doenças semelhantes, provenientes de causas não infecciosas, como trauma, isquemia, queimadura, pancreatite e hemorragia 
(Figura 1). Define-se um quadro de SRIS, quando o paciente manifesta duas ou mais das seguintes condições: hipertermia (temperatura maior que $38^{\circ} \mathrm{C}$ ) ou hipotermia (temperatura menor que $36^{\circ} \mathrm{C}$ ); taquicardia (freqüência cardíaca maior que 90 batimentos/ $\min )$; taquipnéia (freqüência respiratória maior que 20 respirações/ min ou $\mathrm{PaCO}_{2}$ menor que $32 \mathrm{mmHg}$ ) e contagem das células brancas totais sangüíneas maior que

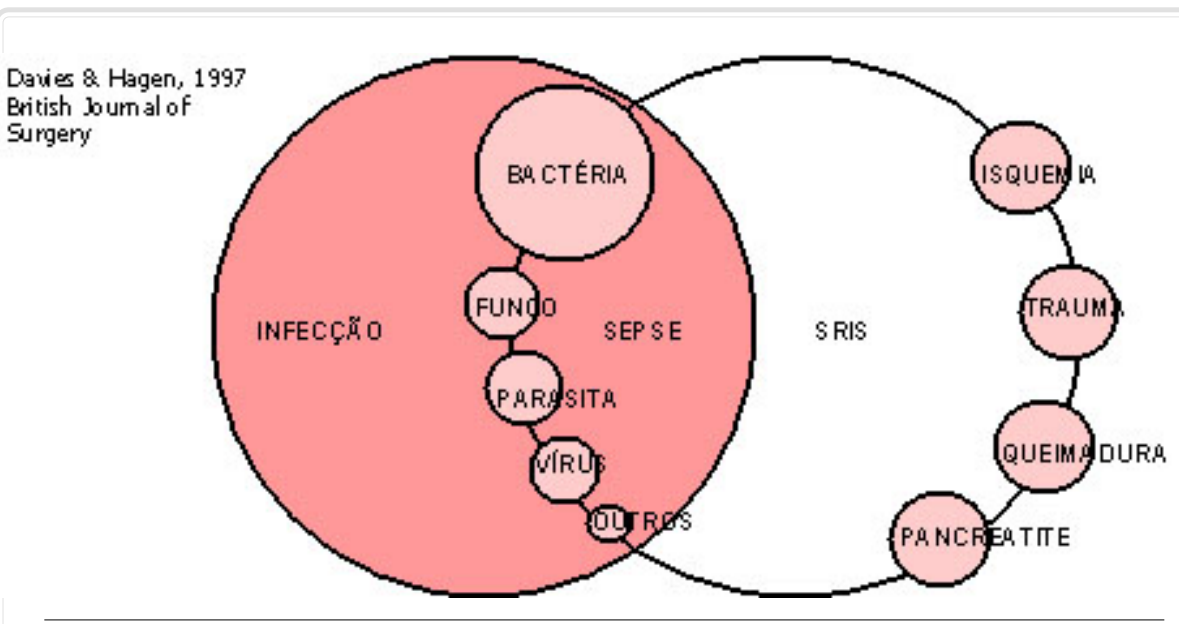

Figura 1: Definições de SRIS, Sepse e Infecção. $12.000 / \mathrm{mm}^{3}$ ou menor que $4.000 / \mathrm{mm}^{3}$ ou com mais de $10 \%$ de formas imaturas $^{(2)}$. Uma das sequielas da SRIS ou da sepse, que pode vir a surgir, é a síndrome da disfunção de múltiplos órgãos (SDMO), que acomete cerca de 30\% dos pacientes com sepse, enquanto quase todos desenvolvem disfunção de um órgão ${ }^{(3)}$. O choque séptico é um agravamento do quadro de sepse, caracterizado por acentuadas alterações hemodinâmicas: 1) hipotensão definida por uma pressão arterial média (PAM) menor que $90 \mathrm{mmHg}$ ou uma redução de $40 \mathrm{mmHg}$ da PAM basal, não responsiva à reposição de líquido e resistente a agentes vasoconstritores; 2) perfusão anormal de órgãos e tecidos, procedente da vasodilatação e vasoconstrição em pequenos vasos; 3) diminuição da resistência vascular sistêmica e 4) aumento da frequiência cardíaca (100 batimentos/ min ou mais) ${ }^{(4)}$.

\section{FATORES DESENCADEADORES DA SEPSE}

As manifestações clínicas da sepse e do choque séptico, tais quais febre, hipercoagulação e hipotensão periférica, derivam da liberação sistêmica de mediadores inflamatórios pelas células de defesa e células endoteliais. Os fatores desencadeadores da ativação celular e da cascata de eventos plasmáticos são componentes da parede celular desses organismos, como o ácido lipoteicóico (LTA) e peptideoglicanos, derivados de bactérias gram-positivas (exotoxinas), ou o lipopolissacarídeo (LPS), no caso de bactérias gramnegativas (endotoxinas) ${ }^{(5)}$. O LPS e as exotoxinas são liberados normalmente, durante a replicação bacteriana e como conseqüência de sua morte, devido à lise da parede celular. O LPS é uma molécula composta por uma cadeia polissacarídica, hidrofílica, a qual se divide em antígeno $\mathrm{O}$ e o centro ("core"), e a porção do lipídio $\mathrm{A}^{(4)}$. O lipídio A é uma região conservada do LPS, é ligado a uma porção polissacarídea, sendo o principal responsável pela toxicidade da endotoxina ${ }^{(6)}$. A importância do LPS, como fator desencadeador da sepse, foi demonstrada após sua administração em humanos sadios, com reprodução de alterações hemodinâmicas, observadas em pacientes com sepse e em modelos experimentais ${ }^{(7)}$.

\section{MODELOS EXPERIMENTAIS DE SEPSE}

Dentre os modelos experimentais, utilizados no estudo de sepse, distinguem-se os relacionados a seguir.

\section{a) Administração endovenosa da bactéria viva ou de componentes microbianos}

A administração endovenosa de LPS (endotoxemia) ou de bactérias como a E. Coli (bacteriemia) é amplamente utilizada para o estudo da sepse, por mimetizar vários efeitos observados em pacientes com sepse e choque séptico, como, por exemplo, as alterações hemodinâmicas e cardiovasculares, diminuição do débito urinário, redução da perfusão tissular, hiporresponsividade a agentes vasoconstritores, coagulação intravascular disseminada e a produção de grandes quantidades de citocinas na circulação. Além disso, é um modelo prático e reproduzível em muitas espécies animais, como em ratos ${ }^{(8)}$, camundongos ${ }^{(9)}, \operatorname{coelhos}^{(10)}$, cachorros $^{(11)}$ e primatas ${ }^{(12)}$, inclusive em humanos ${ }^{(13)}$. Entretanto, a incidência de sepse e choque séptico, na 
clínica, devido à entrada de uma grande quantidade de LPS ou bactéria na circulação (bolus) é muito pequena, levantando a questão se realmente esse modelo mimetiza a evolução de um quadro de sepse.

\section{b) Administração intraperitoneal da bactéria viva ou de componentes microbianos}

Esse modelo, também, é muito utilizado para o estudo da sepse, pois reproduz os sinais observados na doença, apresentando reprodutibilidade, como observado no modelo de endotoxemia. Além disso, a administração de LPS ou bactérias na cavidade peritoneal se aproxima mais de um quadro de sepse observado na clínica, pois o processo se inicia a partir do foco infeccioso ou da disseminação de LPS administrados na cavidade peritoneal, e não diretamente na circulação ${ }^{(14,}$ 15). Mas, ainda assim, o início do processo ocorre de forma muito rápida, e não gradativa, como acontece na maioria dos casos clínicos.

\section{c) Modelos de injúria do intestino com consecu- tiva liberação de flora microbiana}

- Ligação e perfuração do ceco (cecal ligation and puncture - CLP),

- Introdução de cateter no cólon ascendente (colon ascendens stent peritonitis - CASP).

O modelo de injúria com liberação da flora bacteriana é o que mais se assemelha ao quadro de sepse em humanos, decorrente de traumas com perfurações das alças intestinais, colite ou peritonite pósoperatória. Nesse modelo, após a perfuração da parede intestital, ocorre a liberação gradativa do conteúdo cólico para a cavidade peritoneal, induzindo peritonite, a qual pode evoluir para um quadro de sepse e choque séptico.

Apesar de esse modelo experimental estar próximo a um quadro clínico, e, por isso, ser um modelo mais interessante para o estudo da sepse, a maioria dos estudos em sepse experimental baseia-se em modelos nos quais a bactéria ou o LPS são administrados i.v ou i.p. Dados da literatura mostram que a patogênese da sepse, causada pela administração de LPS ou de bactérias i.v., difere daquela induzida por um foco infeccioso, como acontece em uma peritonite ${ }^{(16,17,18)}$. A diferença dos resultados obtidos entre esses modelos é devida à quantidade do estímulo, ao local e à forma de administração (em bolus ou liberação gradativa), induzindo uma cinética distinta de liberação dos mediadores inflamatórios ${ }^{(14,19,20)}$.

A partir desses fatos, a utilização do modelo CLP para o estudo da sepse, descrito inicialmente por
Wichtermann et al., em $1980^{(21)}$, e Baker et al., em $1983^{(22)}$, apresenta maior relevância para a compreensão da evolução da doença.

\section{MEDIADORES INFLAMATÓRIOS, EN- VOLVIDOS NA SEPSE}

A atividade biológica das endotoxinas e exotoxinas é decorrente da ativação de sistemas séricos, de células residentes, do endotélio e dos leucócitos. Conseqüentemente, ocorre síntese e/ou liberação de mediadores endógenos (citocinas, radicais reativos do oxigênio e do nitrogênio e mediadores lipídicos), sendo a ativação das células inflamatórias o fator predominante para o desenvolvimento da sepse ${ }^{(6)}$.

\section{a) Citocinas}

Dentre os mediadores endógenos, liberados, primeiramente, pelas células residentes e, posteriormente, pelas células recrutadas para o foco infeccioso, as citocinas desempenham um papel primordial na resposta do hospedeiro. Citocinas como a interleucina-1 (IL-1), o fator de necrose tumoral (TNF- $\alpha$ ), a IL-8 e as demais quimiocinas promovem o recrutamento de leucócitos para o sítio inflamatório, bem como sua ativação com conseqüente aumento da atividade microbicida, sendo a resposta local fundamental para o controle da infecção. A evolução de uma infecção localizada para um quadro sistêmico caracteriza-se pela presença de citocinas pró-inflamatórias na circulação e ativação das células circulantes, podendo, também, haver a presença de bactérias, endotoxinas ou exotoxinas. As mesmas citocinas encontradas no local da infecção estão presentes na circulação e provêm do tecido e/ou do endotélio ou, ainda, dos leucócitos sangüíneos. Esse fato é responsável pela maioria das alterações fisiopatológicas, observadas em um quadro clínico de sepse e em modelos experimentais.

O TNF- $\alpha$ é tido como um mediador importante na sepse a partir de várias evidências: a) é a primeira citocina que aparece na circulação, na sepse experimental e em humanos; b) a administração de TNF i.v., em animais, induz uma síndrome com as características da sepse, c) o tratamento com anticorpo anti-TNF protege contra os efeitos letais da endotoxina, em vários modelos animais ${ }^{(23)}$. Todavia, vale a pena ressaltar que a importância do TNF, na evolução da sepse, difere entre os modelos estudados como, por exemplo, na peritonite induzida em ratos, em que o tratamento com anticorpo anti-TNF não alterou a sobrevida dos animais ${ }^{(16)}$. Por outro lado, o anticorpo contra o 
TNF- $\alpha$ aumenta a sobrevida dos animais na endotoxemia, ao passo que seu efeito é deletério na CLP não letal, aumentando a taxa de mortalidade. Esses resultados sugerem que, na endotoxemia, o TNF- $\alpha$ estaria envolvido no agravamento da sepse, enquanto, na CLP, a citocina seria importante para a resposta de defesa do indivíduo ${ }^{(24,25)}$. Esses dados sugerem que essa citocina não parece ser crítica para todas as formas de sepse, como, também, não é o único mediador envolvido nesse processo. Assim, a relevância do TNF- $\alpha$, na sepse, varia entre os modelos utilizados, a quantidade do estímulo (LPS, bactéria ou conteúdo cólico) e o local do inóculo.

Além disso, a utilização de anticorpos anti TNF- $\alpha$, em ensaios clínicos, tem demonstrado resultados pouco satisfatórios; pacientes com sepse severa e choque séptico, que receberam infusão do anticorpo monoclonal anti TNF- $\alpha$, não tiveram diminuição na mortalidade avaliada, durante o período do estudo (28 dias). No entanto, quando a avaliação do tratamento com o anticorpo é realizada em um grupo mais homogêneo de pacientes, como, por exemplo, nos que apresentaram altos níveis de TNF- $\alpha$, no início do estudo, ou, ainda, só nos pacientes com choque séptico, observa-se que eles tiveram aumento na sobrevida e diminuição dos níveis séricos de TNF- $\alpha^{(26,27,28)}$.

A participação da IL-1, na fisiopatologia da sepse, foi bem demonstrada pela observação de que um antagonista do receptor para essa citocina (IL-1ra) reduziu a letalidade causada pela administração de endotoxina ${ }^{(29)}$ ou E. Coli ${ }^{(30)}$, em coelhos. Além disso, a IL-1, quando administrada i.v., em humanos, mimetiza sintomas verificados em pacientes com choque séptico, como a hipotensão, febre e outros. Também foi demonstrado que camundongos deficientes na produção da enzima conversora da IL-1 $\beta$ (responsável pela clivagem da pró-IL-1 $\beta$ em IL-1 $\beta$ madura) não produzem essa citocina após estimulação com LPS e são resistentes ao choque séptico ${ }^{(31)}$. Nos estudos de sepse, em humanos, o aumento dos níveis plasmáticos de TNF- $\alpha$ e IL-1 $\beta$ foi correlacionado com o grau de severidade da doença ${ }^{(32)}$, detectando-se níveis mais elevados nos casos fatais $^{(33)}$. Cabe ressaltar que a IL-1 $\beta$ e o TNF- $\alpha$ estimulam a liberação sistêmica de IL-6 e IL-8, amplificando, desse modo, a resposta inflamatória ${ }^{(28)}$.

A IL-8 é uma proteína de baixo peso molecular, pertencente à subfamília $\alpha$ das quimiocinas (citocinas quimiotáxicas para leucócitos). É um potente agente quimiotáxico e ativador de neutrófilos, capaz de aumentar a resposta inflamatória pela indução da liberação de radicais livres e enzimas proteolíticas. $\mathrm{O}$ au- mento da produção da IL-8 foi demonstrado na sepse, em humanos, ou na sepse induzida experimentalmente por administração intravenosa de endotoxina ou bactérias. Em macacos, após administração intravenosa de $E$. Coli, LPS, TNF- $\alpha$ ou de IL- $1 \beta$, houve um aumento dos níveis plasmáticos de IL-8, sendo o pico de aparecimento relativamente tardio, após o TNF- $\alpha$ e a IL-1 ${ }^{(34,35)}$. Por outro lado, a presença de IL-8, no foco infeccioso, é de extrema importância, devido à grande habilidade em induzir o recrutamento de neutrófilos para o local, contribuindo para a eliminação dos microorganismos ${ }^{(36)}$.

O aumento dos níveis plasmáticos da IL-6 é simultâneo ao aparecimento da IL-8 ${ }^{(35)}$. A IL-6 participa, principalmente, na indução da febre e na produção, pelo fígado, de proteínas de fase aguda. Apesar de não estar clara a relevância de seus efeitos na sepse, essa citocina é a que apresenta melhor correlação com a mortalidade, em modelos experimentais e em pacientes com sepse, isto é, quanto maior os níveis plasmáticos de IL-6, maior a probabilidade de o paciente morrer ${ }^{(24,26,27,34)}$. A liberação de IL-8 e de IL-6 é dependente da produção de TNF, pois a neutralização dessa citocina com anticorpos específicos diminui os níveis plasmáticos da IL-6 e IL-8 ${ }^{(35)}$.

Em resumo, há diversos relatos, na literatura, demonstrando a correlação existente entre o aumento da produção de citocinas pró-inflamatórias e o índice de mortalidade na sepse, em humanos e em modelos experimentais, por administração sistêmica de LPS ou bactérias.

Um dado interessante, observado na sepse, através dos modelos experimentais, é que a presença de altos níveis de citocinas na circulação, como o TNF- $\alpha$ e IL-8, reduz o recrutamento de neutrófilos para o sítio injuriado, fato observado na endotoxemia ${ }^{(37)} \mathrm{e}$, também, na CLP (trabalho submetido). Em modelo, no qual não existe foco infeccioso, como na endotoxemia, a falência da migração de neutrófilos não acarreta grandes consequiências, já que o acúmulo de neutrófilos, em tecidos não infectados, é extremamente lesivo devido à ativação dessas células e à liberação de enzimas proteolíticas e de radicais livres que são altamente reativos. Já em modelos, nos quais há presença de um foco infeccioso, como na CLP, a falência da migração de neutrófilos para o sítio é extremamente prejudicial para a resolução do processo, uma vez que essas células são fundamentais para a eliminação dos microorganismos, principalmente bactérias. Dessa forma, ocorre proliferação e disseminação do agente infeccioso no organismo, evoluindo o quadro de sepse para 
o choque séptico e a invariável morte do indivíduo. Nosso laboratório demonstrou que existe uma correlação entre a falência da migração de neutrófilos para o foco infeccioso e a alta mortalidade observada no choque séptico, modelo de CLP; entretanto ainda não demonstramos quais os mediadores que participam, efetivamente, desse processo.

Paralelamente à liberação das citocinas pró-inflamatórias, o organismo responde a agentes infecciosos, liberando citocinas antiinflamatórias como IL-4, IL-10, IL-13, TGF- $\beta$, o antagonista de receptor da IL1 (IL-1ra), além de receptores solúveis para IL-1 (IL$1 \mathrm{R}$ tipo II), TNF- $\alpha$, IL-6 e outros. Esses mediadores são encontrados tanto nos soros como em exsudatos obtidos de pacientes com sepse ou em modelos experimentais, e parecem tanto contrabalançar as ações dos mediadores pró-inflamatórios, através da redução da síntese e da liberação desses mediadores, quanto antagonizar seus efeitos ${ }^{(28)}$. Em modelos de endotoxemia, em camundongos, a IL-10 apresenta um efeito protetor que estaria relacionado à inibição da produção de TNF- $\alpha^{(38,39,40)}$. Em chimpanzés, o TNF parece atuar como mediador da produção de IL-10, sugerindo um mecanismo de regulação negativa da própria liberação do $\mathrm{TNF}^{(41)}$. Em paralelo, a IL-10, também, apresenta um papel regulatório sobre outras citocinas, como, por exemplo, a IL-2 e INF- $\gamma^{(42)}$. Por outro lado, a produção e liberação excessiva de mediadores antiinflamatórios é prejudicial à resposta do organismo contra o agente invasor, pois inibe a liberação dos mediadores fundamentais para o recrutamento e ativação das células da resposta inflamatória e imunes. Com base nos resultados apresentados até então, pode-se sugerir que a exacerbação da liberação de citocinas pró ou antiinflamatórias está fortemente relacionada com a severidade e mortalidade na sepse, sendo primordial o equilíbrio desses mediadores para a resolução dessa doenças ${ }^{(19)}$.

Além das citocinas, os mediadores lipídicos (como as prostaglandinas, PAF e leucotrienos) e os componentes plasmáticos (cininas, fatores da coagulação e complemento) também participam do desenvolvimento da sepse, induzindo, dentre outros efeitos, a migração e ativação celular, a vasodilatação, a agregação plaquetária e o aumento de permeabilidade vascular.

\section{b) Óxido nítrico}

O acúmulo de leucócitos e sua ativação pelos mediadores descritos acima levam à formação de espécies reativas de oxigênio e nitrogênio, como ânion superóxido $\left(\mathrm{O}_{2}^{-}\right)$e óxido nítrico (NO), respectivamente. Além dos seus efeitos próprios, esses radicais também podem reagir entre si, produzindo o peroxinitrito $\left(\mathrm{ONOO}^{-}\right)$, um potente agente oxidante ${ }^{(43)}$. O NO é formado a partir da oxidação do nitrogênio terminal do aminoácido L-arginina pela ação da enzima sintase de óxido nítrico (nitric oxide sinthase - NOS). Existem, pelo menos três, isoformas bem definidas dessa enzima, que são: 1) NOS neuronal (NOSn ou NOS I), descrita inicialmente nos neurônios; 2) NOS induzida (NOSi ou NOS II), presente em leucócitos ativados e 3) NOS endotelial (NOSe ou NOS III), primeiramente encontrada nas células endoteliais (o NO foi, primeiramente, descrito por Furchgott, 1980 ${ }^{(44)}$; Knowles \& Moncada, 1994 ${ }^{(45)}$.

Dentre seus inúmeros efeitos, o NO é uma molécula que apresenta atividade vasodilatadora e citotóxica, e sua produção, quando desregulada, contribui significativamente para a patogênese da sepse e para o choque séptico ${ }^{(46)}$. Hoje está claro que o NO responsável pela maioria dos efeitos patológicos na sepse é procedente da atividade da enzima induzida, que é capaz de produzir NO em concentração micromolar, em comparação à enzima constitutiva que produz esse mediador apenas em concentração nanomolar. A NOS II é induzida por citocinas, como TNF- $\alpha$, IL-1- $\beta$, interferon- $\alpha, \beta$ e $\gamma$ (IFN- $\alpha, \beta, \gamma)$, liberadas tanto durante um processo inflamatório, ou infeccioso $^{(47)}$ como durante a endotoxemia ${ }^{(48)}$. A origem do NO, no processo inflamatório, não é clara, podendo decorrer das células endoteliais, neutrófilos e macrófagos $^{(48)}$.

O NO faz parte do arsenal utilizado pelos leucócitos para suas atividades microbicidas, pois é extremamente reativo e forma complexos com hemeproteínas, inativa proteínas que possuem centros de ferro/ sulfato e produz produtos como nitrosotiol e peroxinitrito $\left(\mathrm{ONOO}^{-}\right)^{(43)}$. Macrófagos ativados com IFN- $\gamma$ apresentam atividade microbicida intracelular, por mecanismos dependentes da síntese de NO, como demonstrado para a infecção por Trypanosoma rruzi $^{(49)}$. Fierro et al. $1996^{(50)}$, demonstraram em experimentos in vitro que o efeito microbicida dos neutrófilos contra Candida albicans acontece, pelo menos, em parte, por meio da liberação de NO. Esses dados já nos mostram a importância da produção de NO durante a sepse e choque séptico, como uma ferramenta fundamental para a eliminação dos microorganismos pelas células de defesa, limitando a disseminação desses agentes e evolução do processo. 
Entretanto, ao mesmo tempo que o NO é importante para a atividade microbicida, ele é um dos responsáveis pela hipotensão observada durante o choque séptico, visto que inibidores seletivos da NOSi são capazes de reverter o quadro ${ }^{(46)}$. O NO induz relaxamento do músculo liso vascular, acarretando queda da pressão arterial. A hipotensão observada no choque séptico é resistente a agentes vasoconstritores, e esse fato é, também, devido à ação do NO. Porém, o mecanismo de ação pelo qual o NO causa hiporresponsividade a agentes vasoconstritores ainda não está claro.

Na sepse experimental, causada pela administração intravenosa de endotoxina ou bactérias, na qual se observa aumento da produção de NO, camundongos deficientes para a NOSi mostraram-se mais resistentes aos efeitos letais do modelo. São dados que apontam para o NO, produzido pela NOSi, como um mediador importante na mortalidade provocada pelo LPS $^{(51)}$.

Paralelamente a isso, há evidências de que o NO modula a adesão de leucócitos ao endotélio, visto que a inibição da NOS por L-NAME e L-NMMA resulta no aumento na aderência dos leucócitos às vênulas pós-capilares ${ }^{(52)}$. O fato foi, primeiramente, demonstrado em preparações in vivo de vênulas do mesentério de gatos, nos quais a perfusão com L-NMMA ou LNAME aumentou a adesão dos leucócitos, sendo esse efeito revertido pelo anticorpo monoclonal anti CD18 ${ }^{(53)}$. O mesmo efeito foi observado, também, in vitro, em ensaio de adesão de neutrófilos a células endoteliais da veia umbilical humana ${ }^{(54)}$.

Corroborando esses dados da literatura, nosso laboratório demonstrou, inequivocamente, que o NO é o efetor fundamental na falência da migração de neutrófilos para um sítio inflamatório ou foco infeccioso, nos modelos de endotoxemia e CLP, visto que inibidores da produção desse mediador preveniram a falência da migração de neutrófilos, induzida pela administração endovenosa de citocinas e pela sepse induzida por CLP.

O mecanismo pelo qual o NO reduz a adesão de leucócitos ao endotélio ainda não está claro. Foi sugerido que esse efeito ocorre devido à habilidade do $\mathrm{NO}$ em interagir com o $\mathrm{O}_{2}^{-}$liberado pelas células endoteliais, retirando-o do meio. Sabe-se que o $\mathrm{O}_{2}^{-}$pode sofrer uma reação de dismutação, produzindo o peróxido de hidrogênio $\left(\mathrm{H}_{2} \mathrm{O}_{2}\right)$, o qual induz a adesão de leucócitos ao endotélio pela formação de PAF e pelo aumento da expressão de moléculas de adesão (P-selectinas e ICAM-1). Além disso, o $\mathrm{O}_{2}^{-}$estimula os mastócitos a liberarem agentes pró-adesivos (PAF, citocinas), os quais aumentam ainda mais a interação entre células endoteliais e os leucócitos ${ }^{(52,55,56)}$. Logo, o balanço entre a produção de $\mathrm{NO}$ e $\mathrm{O}^{-}$parece ser um determinante essencial na evolução da sepse.

Em adição, Spiecker et al., $1998^{(57)}$ demonstraram uma ação direta do NO, reduzindo a expressão de moléculas de adesão no endotélio pela indução da atividade promotora do Ikappa B-alfa (IאB- $\alpha$ ), um inibidor endógeno do fator de transcrição NF- $\kappa B$ (fator nuclear kappa-B). Esse fator é responsável pela indução gênica de várias moléculas pró-inflamatórias, como as moléculas de adesão, importantes para o rolamento e aderência dos leucócitos às células endoteliais.

$\mathrm{O} \mathrm{NO}$ e os outros mediadores endógenos, já descritos, podem agir de forma seqüencial e/ou antagônica, e a manutenção desse equilíbrio é importante para uma resposta adequada do organismo contra a infecção; uma hiperprodução ou não-produção desses mediadores agrava o quadro de sepse.

\section{CONSIDERAÇÕES FINAIS}

Apesar de grandes avanços terem ocorrido para a compreensão dos mediadores endógenos e seus papéis na resposta inflamatória, durante a sepse, um longo caminho ainda deve ser percorrido para que se entendam os complexos mecanismos fisiopatológicos dessa doença. Está claro, na literatura, que mediadores como TNF- $\alpha$, IL-1 $\beta$, IL-6, IL-8 e NO apresentam papéis fundamentais na sepse, e que os mediadores antiinflamatórios estão presentes, concomitantemente, modulando os efeitos e a liberação dos mediadores inflamatórios. A relação desses mediadores é fundamental para a evolução ou resolução do processo, porém o fator determinante para uma direção, ou para outra, não está claro ainda hoje. Vale a pena ressaltar a participação das citocinas e do NO na migração de neutrófilos e a importância desse evento para a resolução da doença. A falência da migração de neutrófilos, durante o choque séptico, correlaciona-se com a alta mortalidade, e o NO parece ser a molécula efetora no processo de redução da migração. Os modelos experimentais, utilizados para o estudo da sepse, contribuem para um melhor entendimento da evolução da doença, tentando compreender os mecanismos pelos quais os mediadores inflamatórios, envolvidos no processo, desempenham seus papéis, e para que, no futuro, ocorra redução da alta mortalidade decorremte dessa importante entidade clínica. 
BENJAMIM CF. Present understanding of mediators and experimental models of sepsis. Medicina, Ribeirão Preto, 34: 18-26, jan./march2001.

ABSTRACT: The author presents a reviewed description about the inflammatory response during sepsis, viewing the mainly mediators in this process, as cytokines and nitric oxide, and the role of these mediators in the evolution of this disease. This aspect is not so linear and simple, because the importance of each mediators depend on experimental model, animal species and sepsis stage. Furthermore, it will be reviewed the principal experimental models used for the study of sepsis, discussing the advantage of each one and their contribution for the comprehension of the disease. It has no doubt about the importance of all experimental model of sepsis and septic shock, however it is crucial to distinguish the good and bad features about each model, in order to concern successful results for clinic trials.

UNITERMS: Sepsis. Shock, Septic. Endotoxins. Peritonitis. Lipopolysaccharides. Cytokines.

\section{REFERÊNCIAS BIBLIOGRÁFICAS}

1 - QUEZADO ZMN; BANKS SM \& NATANSON C. New strategies for combatting sepsis: the magic bullets missed the mark...but the search continues. Tibtech 13: 56-63, 1995.

2 - BONE RC; GRODZIN CJ \& BALK RA. Sepsis: A new hypothesis for pathogenesis of the disease process. Chest 112: 235-243, 1997.

3 - DAVIES MG \& HAGEN P. Systemic inflammatory response syndrome. Br J Surg 84: 920-935, 1997.

4 - CROWLEY SR. The pathogenesis of septic shock. Heart Lung 25: 124-134, 1996.

5 - SWEET MJ \& HUME DA. Endotoxin signal transduction in macrophages. J Leukoc Biol 60: 8-26, 1996.

6 - GALANOS C \& FREUDENBERG MA. Mechanism of endotoxin shock and endotoxin hypersensitivity. Immunobiology 187: 346-356, 1993

7 - SUFFRADINI AF; FROMM RE; PARKER MM; BRENNER M; KOVACS JÁ; WESLEY RA \& PARRILLO JE. The cardiovascular response of normal humans to the administration of endotoxin. N Engl J Med 321: 280-287, 1989.

8 - MACARTHUR H; WESTFALL TC; RILEY DP; MISKO TP \& SALVEMINI D. Inactivation of catecholamines by superoxide gives new insights on the pathogenesis of septic shock. Proc Natl Acad Sci USA 97: 9753-9758, 2000.

9 - ROVAI LE; HERSCHMAN HR \& SMITH JB. The murine neutrophil-chemoattractant chemokines LIX, KC and MIP-2 have distinct induction kinetics, tissue distributions and tissuespecific sensitivities to glucocorticoid regulation in endotoxemia. J Leukoc Biol 64: 494-502, 1998.

10 - BEM-SHAUL V; SOFER Y; BERGMAN M; ZUROVSKY Y \& GROSSMAN S. Lipopolysaccharide-induced oxidative stress in the liver: comparison between rat and rabbit. Shock 12: 288-293, 1999.

11 - MITAKA C; HIRATA Y; YOKOYAMA K; NAGURA T; TSUNODA $Y$ \& AMAHA K. Beneficial effect of carboxy-PTIO on hemodynamic and blood gas changes in septic shock dogs. Crit Care (London) 1: 45-50, 1997.
12 - HINSHAW LB; EMERSON TEJr; TAYLOR FBJr; CHANG AC; DUERR M; PEER GT; FLOURNOY DJ; WHITE GL; KOSANKE SD; MURRAY CK; XU R ; PASSEY RB \& FOURNEL MA. Lethal sthaphylococcus aureus-induced shock in primates: prevention of death with anti-TNF antibody. J Trauma 33: 568573,1992

13 - VAN DER POLL T; COYLE SM; MOLDAWER LL \& LOWRY SF. Changes in edotoxin-induced cytokine production by whole blood after in vivo exposure of normal humans to endotoxin. J Infect Dis 174: 1356-1360, 1996.

14 - REMICK DG; NEWCOMB DE; GERALD LB \& CALL DR. Comparison of the mortality and inflammatory response of two models of sepsis: lipopolysaccharide vs cecal ligation and puncture. Shock 13:110-116, 2000.

15 - MARTINEAU L \& SHEK PN. Peritoneal cytokine concentrations and survival outcome in an experimental bacterial infusion model of peritonitis. Crit Care Med 28: 788-794, 2000.

16 - BAGBY GJ; PLESSALA KJ; WILSON LA; THOMPSONJJ \& NELSON S. Divergent efficacy of antibody to tumor necrosis factor-alpha in intravascular and peritonitis models of sepsis. J Infect Dis 163: 83-88, 1991.

17 - HADJIMINAS DJ; MCMASTERS KM; ROBERTSON SE \& CHEADLE WG. Enhanced survival from cecal ligation and puncture with pentoxifylline is associated with altered neutrophil trafficking and reduced interleukin-1 beta expression but not inhibition of tumor necrosis factor synthesis. Surgery 116: 348-355, 1994.

18 - VAN DER POLL T; MARCHANT A; BUURMAN WA; BERMAN L; KEOGH CV; LAZARUS DD; NGUYEN L; GOLDMAN M; MOLDAWER LL \& LOWRY SF. Endogenous IL-10 protects mice from death during septic peritonitis. J Immunol 155: 5397-5401, 1995.

19 - WALLEY KR; LUKACS NW; STANDIFORD TJ; STRIETER RM \& KUNKEL SL. Balanced of inflammatory cytokines related to severity and mortality of murine sepsis. Infect Immun 64: 4733-4738, 1996.

20 - ZANTL N; UEBE A; NEUMANN B; WAGNER H; SIEWERT J, HOLZMANN B; HEIDECKE C \& PFEFFER K. Essential role of gamma interferon in survival of colon ascendens stent peri- 
tonitis, a novel murine model of abdominal sepsis. Infect Immun 66: 2300-2309, 1998.

21 - WICHTERMANN KA; BAUE AE \& CHAUDRY IH. Sepsis and septic shock-a review of laboratory models and a proposal. J Surg Res 29: 189-201, 1980.

22 - BAKER CC; CHAUDRY IH; GAINES HO \& BAUE AE. Evaluation of factors affecting mortality rate after sepsis in a murine cecal ligation and puncture model. Surgery 94: 331335, 1983.

23 - VAN DER POLL T \& SAUERWEIN HP. Tumor necrosis factoralpha: its role in the metabolic response to sepsis. Clin Sci 84: 247-256, 1993.

24 - ECHTENACHER B; FALK W; MÄNNEL DN \& KRAMMER PH. Requirements of endogenous tumor necrosis factor/ cachectin for recovery from experimental peritonitis. $\mathbf{J}$ Immunol 145: 3762-3766, 1990.

25 - ZANETTI G; HEUMANN D; GÉRAIN J; KOHLER J; ABBET P; BARRAS C; LUCAS R; GLAUSER MP \& BAUMGARTNER JD. Cytokine production after intravenous or peritoneal gramnegative bacterial challenge in mice. J Immunol 148: 18901897, 1992.

26 - FISHER CJ; OPAL SM; DHAINAUT JF; STEPHENS S; ZIMMERMAN JL; NIGHTINGALE P; HARRIS SJ; SCHEIN RMH; PANACEK EA; VINCENT JL; FOULKE GE; WARREN EL; GARRARD C; PARK G; BODMER MW; COHEN J; Van der LINDEN C; CROSS AS \& SADOFF JC. Influence of na antitumor necrosis factor monoclonal antibody on cytokine levels in patients with sepsis. Crit Care Med 21: 318-327, 1993.

27 - BLACKWELL TS \& CHRISTMAN JW. Sepsis and cytokines: current status. Br J Anaesth 77: 110-117, 1996.

28 - DINARELLO CA. Proinflammatory and anti-inflammatory cytokines as mediators in the pathogenesis of septic shock. Chest 112: 321S-329S, 1997.

29 - OHLSSON K; BJORK P; BERGENFELDT M; HAGEMAN R \& THOMPSON RC. Interleukin-1 receptor antagonist reduces mortality from endotoxin shock. Nature 348: 550-552, 1990.

30 - WAKABAYASHI G; GELFAND JÁ; BURKE JF; THOMPSON RC \& DINARELLO CA. A specific receptor antagonist for interleukin 1 prevents Escherichia Coli-induced shock in rabbits. FASEB J 5: 338-343, 1991.

31 - LI P; YE X; ALLEN H; BANERJEE S; FRANKLIN S; HERZOG L; JOHNSTON C; MCDOWELL J; PASKIND M; RODMAN L; SALFELD J; TOWNE E; TRACEY D; WARDWELL S; WEI FY; WOUNG W; KAMEN R \& SESHA DRI . Mice deficient in II2beta-converting enzyme are deficient in production of mature IL-1beta and resistant to endotoxic shock. Cell 80: 401411, 1995.

32 - CASEY LC; BALK RA \& BONE RC. Plasma cytokine and endotoxin levels correlates with survival in patients with the sepsis syndrome. Ann Intern Med 119: 771-778, 1993.

33 - GARDLUND B; SJOLIN J; NILSSON A; ROLL M; WICKERTS CJ \& WRETLIND B. Plasma levels of cytokines in primary septic shock in humans: correlation with disease severity. J Infect Dis 172: 296-301, 1995.
34 - VAN ZEE KJ; DEFORGE LE; FISHER E; MARANO MA; KENNEY JS; REMICK DG; LOWRY SF \& MOLDAWER LL. IL-8 in septic shock, endotoxemia and after IL-1 administration. J Immunol 146: 3478-3482, 1991.

35 - REDL H; SEHLOG G; CESKA M; DAVIES J \& BUURMAN WA. Interleukin-8 release in baboon septicemia is partially dependent on tumor necrosis factor. J Infect Dis 167: 1464-1466, 1993.

36 - ROT A. Endothelial cell binding of NAP-1/IL-8 role in neutrophil emigration. Immunol Today 13: 291-294, 1992.

37 - TAVARES-MURTA BM; CUNHA FQ \& FERREIRA SH. The intravenous administration of tumor necrosis factor alpha, interleukin 8 and macrophage-derived neutrophil chemotactic factor inhibits neutrophil migation by stimulating nitric oxide production. Br J Pharmacol 124:1369-1374. 1998.

38 - GÉRARD C; BRUYNS C; MARCHANT A; ABRAMOWICZ D; VANDENABEELE P; DELVAUX A; FIERS W; GOLDMAN M \& VELU T. Interleukin 10 reduces the release of tumor necrosis factor and prevents lethality in experimental endotoxemia. J Exp Med 177: 547- 552, 1993.

39 - HOWARD M; MUCHAMUEL T; ANDRADE S \& MENON S. Interleukin-10 protecs mice from lethal endotoxemia. J Exp Med 177: 1205-1209, 1993.

40 - ROGY M.A; AUFFENBERG T; ESPAT NJ; PHILIP R; REMICK D; WOLLENBERG GK; COPELAND III EM \& MOLDAWER LL. Human tumor necrosis factor receptor (p55) and interleukin 10 gene transfer in the mouse reduces mortality to lethal endotoxemia and also attenuates local inflammatory responses. J Exp Med 181: 2289-2293, 1995.

41 - WANIDWORANUN C \& STROBER W. Predominant role of tumor necrosis factor- $\alpha$ in human monocyte IL-10 synthesis. J Immunol 151: 6853-6861, 1993.

42 - FLORQUIN S; AMRAOUI Z; ABRAMOWICZ \& GOLDMAN M. Systemic release and protective role of endogenously synthesized nitric oxide in Staphylococal enterotoxin B-induced shock in mice. J Immunol 153: 2618-2623, 1994.

43 - HAMPTON MB; KETTLE AJ \& WINTERBOURN CC. Inside the neutrophil phagosome: Oxidants, myeloperoxidase, and bacterial killing. Blood 92: 3007-3017, 1998.

44 - FURCHGOTT RF \& ZAWODZKI JV. The obligatory role of endothelial cells in the relaxation of arterial smooth muscle by acetylcholine. Nature 288(5789): 373-376, 1980.

45 - KNOWLES RG \& MONCADA S. Nitric oxide synthases in mammals. Biochem J 298: 249-258, 1994.

46 - WOLKOW PP. Involvement and dual effects of nitric oxide in septic shock. Inflamm Res 47: 152-166, 1998.

47 - CUNHA FQ; MOSS DW; REES D; LEAL LMC; MONCADA S; CARRIER M; O'DONNELL CA \& LIEW FY. Differential induction of nitric oxide synthase in various organs of the mouse during endotoxemia: role of TNF- $\alpha$ and IL-1 $\beta$. Immunology 81: 211-215, 1994.

48 - MONCADA S \& HIGGS EA. Mechanisms of disease. The Larginine-nitric oxide pathway. N Engl Med 329: 2002-2012, 1993. 
49 - SILVA JS; VESPA JS; CARDOSO MA; ALIBERTI JC \& CUNHA FQ. Tumor necrosis factor alpha mediates resistance to Trypanosoma cruzi infection in mice by inducing nitric oxide production in infected gamma interferon-activated macrophages. Infect Immun 63: 4862-4867, 1995.

50 - FIERRO IM; BARJA-FIDALGO C; CUNHA FQ \& FERREIRA SH. The involvement of nitric oxide in the anti-Candida albicans activity of rat neutrophils. Immunology 89: 295-300, 1996.

51 - WEI X; LI M \& LI R. Altered immune responses in mice lacking inducible nitric oxide synthase. Nature 375: 408-411, 1995.

52 - GRANGER N \& KUBES P. Nitric oxide as inflammatory agent. Methods Enzimol 269: 434-443, 1996.

53 - KUBES P; SUZUKI M \& GRANGER DN. Nitric oxide: an endogenous modulator of leukocyte adhesion. Proc Natl Acad Sci USA 88: 4651-4655, 1991.

54 - NIU X; SMITH CW \& KUBES P. Intracellular oxidative stress induced by nitric oxide synthesis inhibition increases endothelial cell adhesion to neutrophils. Circ Res 74: 1133-1140, 1994.
55 - JOHNSTON B; KANWAR S \& KUBES P. Hidrogen peroxide induces leukocyte rolling: modulation by endogenous antioxidant mechanisms including NO. Am J Physiol 271: H614621, 1996.

56 - NIU X; IBBOTSON G \& KUBES P. A Balance between nitric oxide and oxidants regulates mast cell-dependent neutrophil-endothelial cell interactions. Circ Res 79: 992-999, 1996.

57 - SPIECKER M; DARIUS H; KABOTH K; HÜBNER F \& LIAO JK. Diferential regulation of endothelial cell adhesion molecule expression by nitric oxide donors and antioxidants. J Leukoc Biol 63:732-739, 1998.

Recebido para publicação em 06/07/2000

Aprovado ara publicação em 17/01/2001 